\title{
Integrated PiezoMEMS Actuators and Sensors
}

\author{
Ronald G. Polcawich, Jeffrey S. Pulskamp, Sarah \\ Bedair, Gabriel Smith, Roger Kaul, Chris Kroninger, \\ and Eric Wetzel \\ RF \& Electronics Division \\ US Army Research Laboratory \\ Adelphi, MD, USA \\ ronald.g.polcawich@us.army.mil
}

\author{
Hengky Chandrahalim and Sunil A. Bhave \\ School of Electrical and Computer Engineering \\ Cornell University \\ Ithaca, New York 14853, USA \\ sunil@ece.cornell.edu
}

\begin{abstract}
The ability to combine both actuation and sensing technologies into one unified MEMS fabrication process is enabling for a wide variety of applications including high frequency filters, transformers and mm-scale robotics. This research demonstrates piezoelectric MEMS (PiezoMEMS) devices based on lead zirconate titanate (PZT) thin films including switchable resonators, transformers, and microflight actuators with integrated strain sensors. PiezoMEMS resonators and transformers combine actuation and sensing on a single device platform. Current research on PZT based resonators is focused on optimizing the performance for both high quality factor, $Q$, and low motional resistance. Resonators in the low tens of $\mathrm{MHz}$ can also be utilized as piezoelectric resonant transformers and are predicted to achieve $\mathrm{AC} / \mathrm{DC}$ gains in the range of 2 to 8 . In addition, strain sensors positioned at the cantilever root of PiezoMEMS microflight actuators provide direct feedback of the motional response of the actuated wing.
\end{abstract}

\section{INTRODUCTION}

Piezoelectric MEMS (PiezoMEMS) have begun to make real in roads into a wide variety of device areas including both actuators and sensors. As illustrated in Figure 1, PiezoMEMS can be utilized in a wide variety of categories. Whether its CMOS compatible AIN and $\mathrm{ZnO}$ or high piezoelectric coefficient PZT compounds, PiezoMEMS can be developed for both quasi-static and resonant actuators as well as stress induced and non-stress induced sensors. PZT based devices also include additional benefits related to the ferroelectric response including ferroelectric random access memory (FeRAM) and tunable dielectrics.

One device category that combines the benefits of both actuators and sensors is resonators. In their basic operation, PiezoMEMS resonators operate by transducing an electrical signal into resonant mechanical motion and subsequently transduce that mechanical strain response to an output electrical signal filtered largely by the mechanical frequency response of the electro-mechanical system. In most published PiezoMEMS research, combining both actuators and sensors onto a single unified device or fabrication process is extremely rare. However, a flexible integrated process capable of producing high force actuators along with high sensitivity sensors could be ideal for many applications especially where high functionality and power consumption is critical. One such area of importance is ultra-small scale, mobile unattended sensor systems. This article provides a review of recent advances in PiezoMEMS technology with specific attention to the integration of actuator and sensor devices and the new functionality that this technology can provide in the area of resonant transformers and high frequency filters, switchable filters, and MEMS enabled microflight.

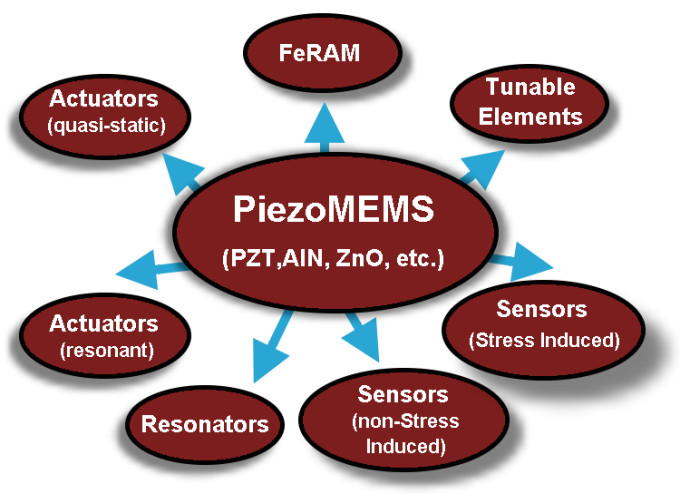

Figure 1: PiezoMEMS device categories

\section{FABRICATION}

Device fabrication was done at the Specialty Electronic Materials and Sensors Cleanroom Facility, U.S. Army Research Laboratory (ARL), Adelphi, MD. The general fabrication process for PiezoMEMS devices at ARL used the following process flow (for more information specific to the 
fabrication of switches, resonators, and mm-scale robotics see [1 - 3]). Starting silicon substrates were first coated by a composite structural layer comprised of silicon dioxide and silicon nitride thin-films deposited by plasma-enhanced chemical vapor deposition (PECVD). Next, a sputtered titanium film was oxided into a textured titanium dioxide film for templating a sputtered (111) platinum thin film to serve as the bottom electrode for the PZT deposition. All PZT thin films were prepared via a chemical solution derived deposition process modified from that outlined in [4]. After the final PZT crystallization anneal, a $1050 \AA$ platinum thin film was sputter deposited directly onto the PZT surface.

The first step in device patterning was to pattern the top platinum electrode with the argon ion-milling (see Figure 2). This etch was then followed by an additional ion-milling of the PZT and bottom electrode features. To open access to the bottom electrode, a via is patterned via ion-milling using an endpoint etch to stop on the platinum layer. After ionmilling, a quick wet etch was used to clean up any remaining PZT related compounds on the bottom platinum electrode. The actuator or sensor structure was then further defined by patterning the composite structural layer with a reactive ion etch to provide access to the silicon device layer for the eventual release etch. A titanium / gold bi-layer was then deposited with electron beam evaporation and patterned via liftoff to define co-planar waveguide transmission lines, contact structures, and anchor features required for gold air bridges. A photo-resist sacrificial layer was then patterned and cured and was followed by the deposition and lift-off of 2 $\mu \mathrm{m}$ gold air-bridge features. If required, the device layer silicon or the handle wafer on silion-on-insulator wafers were patterned and etched via silicon deep reactive ion etching. In the final process step, a $\mathrm{XeF}_{2}$ etch is used to either etch the device layer or regions of the handle wafer in order to create a released PiezoMEMS device.
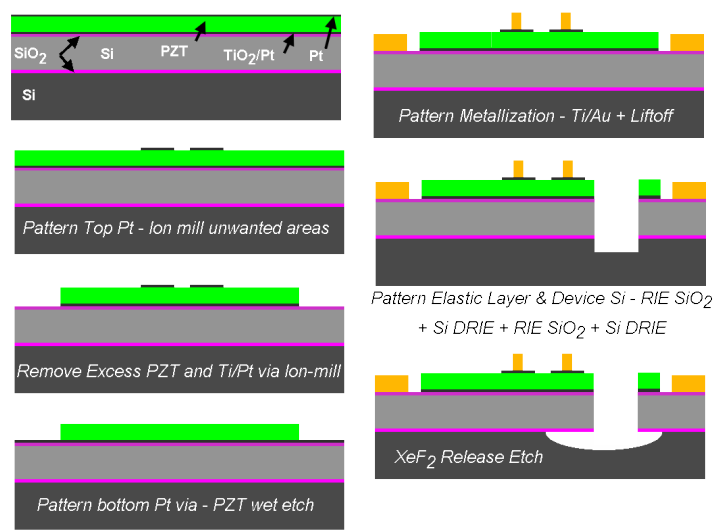

$+\mathrm{SiDRIE}+\mathrm{RIES \textrm {SO } _ { 2 }}+\mathrm{Si}$ DRIE

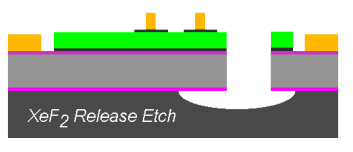

Figure 2: Process flow schematic highlighting the critical steps in device fabrication including ion-milling of the top Pt, PZT and

$\mathrm{TiO}_{2} / \mathrm{Pt}$ layers, a wet etch to open a via to the bottom $\mathrm{Pt}$, evaporation of a contact metallization ( $\mathrm{Ti} / \mathrm{Au})$, patterning the elastic layer and SOI features, and a $\mathrm{XeF}_{2}$ step. For additional information on air bridge processing and sidewall protection of the device layer Si see [2] and [5].

\section{RESULTS}

\section{A. Resonator-Type Devices}

The two most important aspects of resonator-type devices are the mechanical quality factor, $Q$, and the motional resistance of the device in a circuit. To optimize these parameters, PiezoMEMS resonators and resonant transformers have been designed and fabricated using a PZT-on-SOI technology where the single crystal silicon can provide a low loss resonant structure and the PZT can provide a high efficiency electrical-to-mechanical and mechanical-toelectrical converter. Additionally, the integration of PZT thin films single crystal silicon features allows a tradeoff between steep-walled narrow-bandwidth filters, low- $Q$ wide-bandwidth filters, linearity and center frequency agility. Figure 3 illustrates the key features of these devices which include input and output electrodes and tethers to support the devices mechanically and provide electrical connections to the resonator. Rather than discussing the RF performance of these resonators (additional information can be found in $[2,6]$ ), the focus here will be on their use as resonant transformers that are being evaluated as the passive component in a high switching frequency $(>10 \mathrm{MHz})$ power converter architecture. Thin film piezoelectric transformers offer a viable alternative to magnetic transformer technologies, where magnetic materials limit the performance at high frequencies. An array of resonant piezoelectric transformers impedance matched to various loads is envisioned providing an ultra-miniature power conditioning unit. Electrodes may be lithographically defined for load impedance matching. Figure 4 highlights the predicted $\mathrm{AC} / \mathrm{AC}$, load dependent performance for a $20 \mathrm{MHz}$ resonant transformer with a $50 \mathrm{ohm}$ motional resistance in the matched input / output electrode case. Predicted AC/DC gain ratios of 2 to 8 are anticipated for varying load resistances and achieving efficiencies in the range of 29 to $77 \%$. Power handling performance of a representative device illustrated in Figure 5 resulted in a measured device power density greater than $10 \mathrm{~W} / \mathrm{mm}^{3}$ at $51 \mathrm{MHz}$ [7]. This technology (with improvements) should provide a viable alternative to high frequency, micro-machined magnetic transformers which are, at the least, 2 orders of magnitude larger than thin-film, piezoelectric resonant transformers.

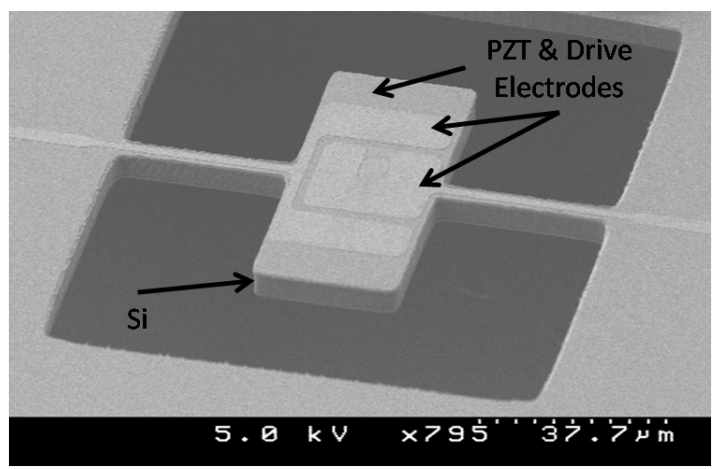

Figure 3: PiezoMEMS resonators and/or resonant transformers utilizing a PZT-on-SOI technology. 


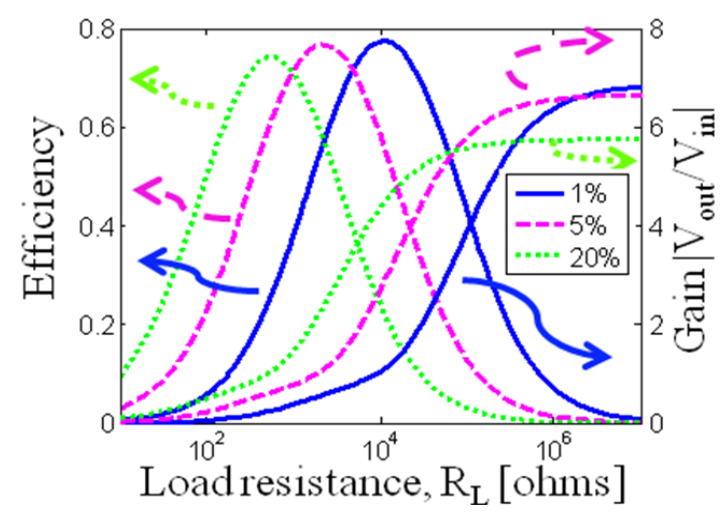

Figure 4: Predicted AC-AC and AC-DC performance of a PiezoMEMS resonant transformer.

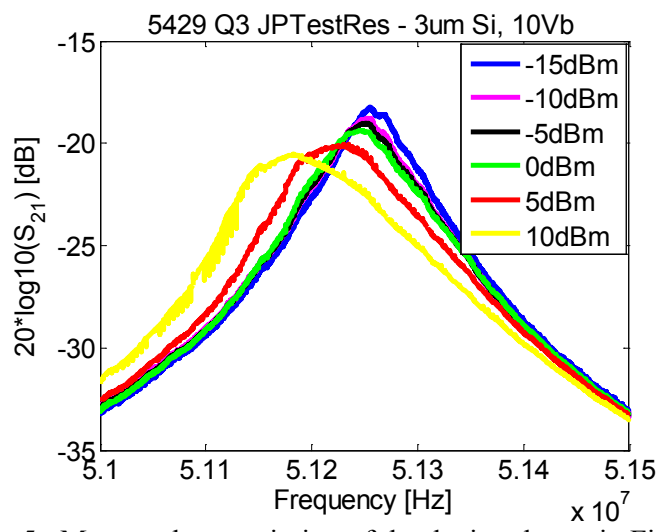

Figure 5: Measured transmission of the device shown in Figure 3 at varying power levels.

\section{B. Combining Actuator-Type With Resonator-Type Devices}

Channel select filtering is highly sought after for future radio and cellular phone applications. A critical component for these applications is the ability to reliably switch between different bandpass filters. Ideally, these functions can be provided by one device so as to reduce the size and weight of the overall system. Using a single pole dual throw (SP2T) architecture similar to that reported in [8], two normally-open, ohmic contact, series switches were used to select between two contour mode mechanically coupled PZT-on- SOI high quality factor filters (see Figure 6) [9]. With switches in the offstate (i.e. 0 volts applied), the output from either the left or right filters exhibited a flat S21 response (into $50 \mathrm{Ohms}$ ) with a magnitude of $-60 \mathrm{~dB}$. As illustrated in Figure 7, every time a switch closes (using 7 volt actuation) the ring up response from the filter can be detected at the output terminal. The filters in this initial device demonstration exhibited out-ofband rejection of nearly $-30 \mathrm{~dB}$ and a $50 \Omega$ terminated insertion loss of $-17 \mathrm{~dB}$ and $-24 \mathrm{~dB}$ (as measured from the minimum insertion loss) with the application of a 10 volt DC bias. Similar filter responses were observed in the integrated filters (add $0.4 \mathrm{~dB}$ insertion loss) as in the standalone filters fabricated on the same wafer. The similar performance between individual filters and filters integrated with SP2T switches suggest obtaining improved device performance is a matter of updating the filter design, which is currently in progress. The integration of low voltage switches and voltage tunable PiezoMEMS filter arrays should provide a drop-in solution for frequency-agile channel selectivity.

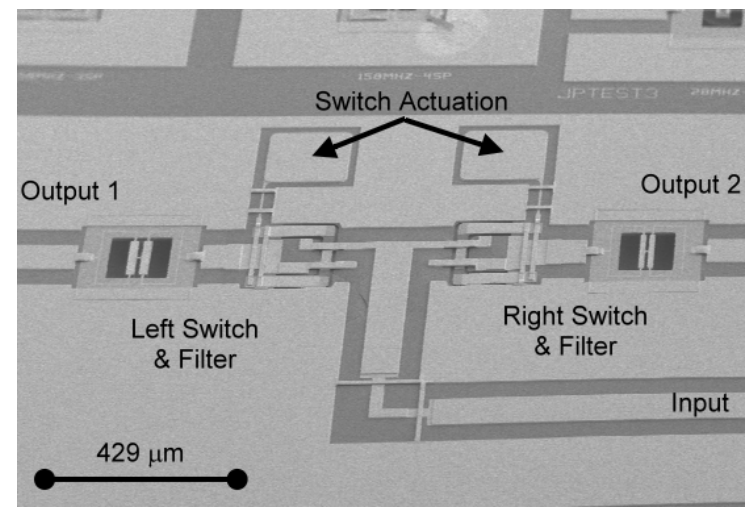

Figure 6: SEM of an integrated single pole dual throw PZT MEMS switch and two PZT contour mode filters (from [9]).

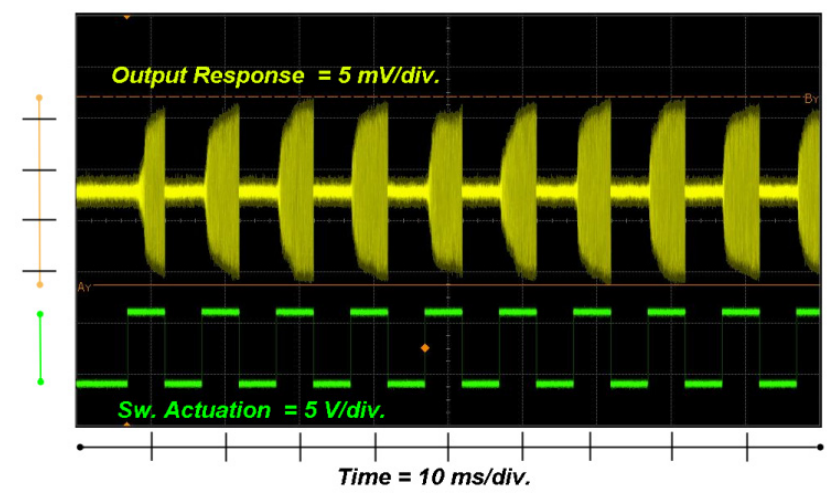

Figure 7: Time domain measurements of the switch and filter highlighting the $7 \mathrm{~V}$ switching actuation pulses and the ring up response of the filter with the switch in the on-state.

\section{Combining Actuator-Type With Stress-Induced Sensor- Type Devices}

For mm-scale robotics, piezoelectric actuators represent an enabling technology for both ground and air mobile platforms at the mm-scale. Combining sensors either for proprioception with feedback in actuator control or exteroception for providing sensory perception (i.e. acoustic, infrared, optical, etc) can aid in the utility of these robotic platforms. As illustrated in Figure 8, PiezoMEMS actuators have been designed to investigate flapping microflight at the mm-scale [10]. In the dual-wing platform, two independent actuators were utilized to generate both flap and angle-of-attack in a microwing while small PZT strain sensors were integrated into the anchor attachment of the wing (see Figure 9). These two degree-of-freedom designs have enabled stroke amplitudes in excess of 84 degrees under resonant drive at about $3.4 \mathrm{~V}$. Increasing the drive voltages for stroke, the wings have achieved in excess of 120 degrees. Quasi-static displacements 
of 36 degrees have been measured for pitch. The strain sensors have been designed as high aspect ratio (length/width) structures and have demonstrated responses in the range of hundreds of millivolts with the wing operating near resonance with a flap amplitude in excess of 20 degrees. These direct wing measurements can be used to enhance lift and real-time flight control strategies of the microflight platforms.

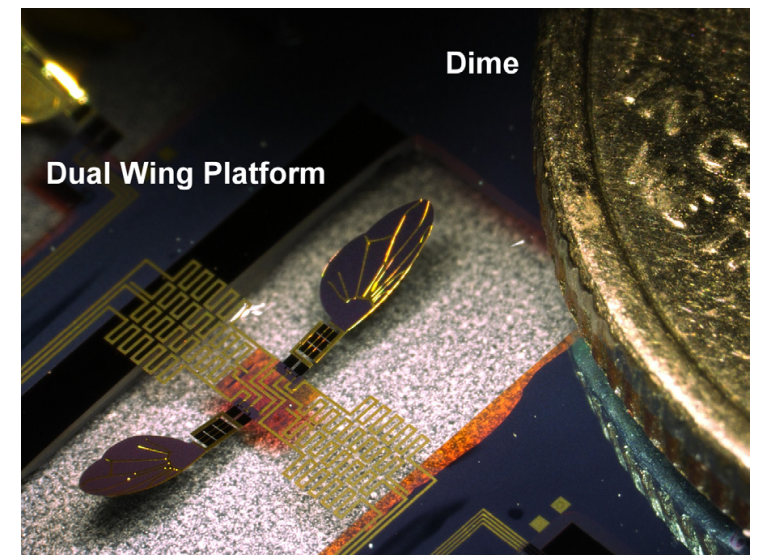

Figure 8: A dual-wing platform using PZT actuators to investigate MEMS enabled microflight.

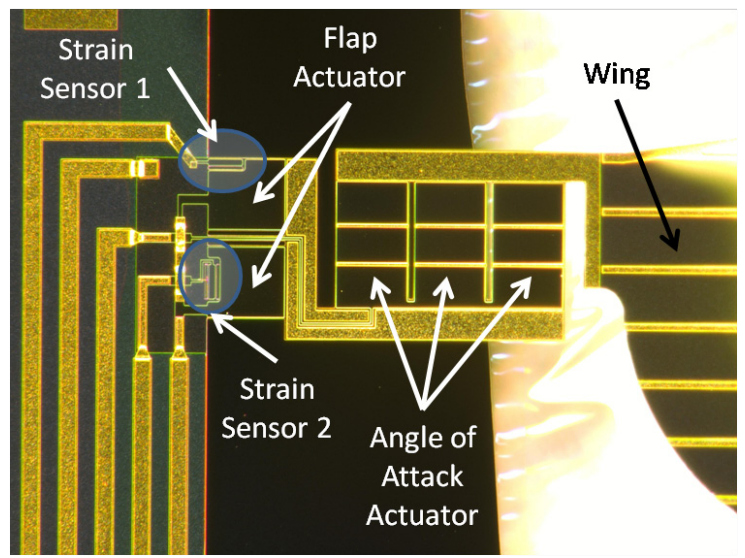

Figure 9: PiezoMEMS enabled microflight actuators (flap and angle-of-attack) with two integrated strain sensors at the root attachment of the flap actuator.

\section{CONCLUSION}

This article provides a brief review of recent advances in PiezoMEMS technology using PZT thin films for both actuation and sensing. The integration of actuator and sensor devices provides new functionality that PiezoMEMS technology can provide in the area of resonant transformers and high frequency filters, switchable filters, and MEMS enabled microflight for mobile unattended sensor platforms.

\section{ACKNOWLEDGMENT}

The authors would like to thank Joel Martin, Richard Piekarz, and Brian Power for their hard work and dedication in the fabrication of the PiezoMEMS Devices.

\section{REFERENCES}

[1] R. Polcawich, J. Pulskamp, D. Judy, P. Ranade, S. Trolier-McKinstry, and M. Dubey, "Surface Micromachined Microelectromechancial Ohmic Series Switch Using Thin-Film Piezoelectric Actuators," IEEE Transactions Microwave Theory and Techniques, Vol. 55, pp. 2642 2654, Dec 2007.

[2] H. Chandrahalim, S.A. Bhave, R.G. Polcawich, J. Pulskamp, D. Judy, R. Kaul, and M. Dubey, "Influence of Silicon on Quality Factor, Motional Impedance and Tuning Range of PZT-Transduced Resonators," Solid State Sensor, Actuator and Microsystems Workshop, Hilton Head Island, South Carolina, pp. 360-363, 2008.

[3] J.R. Bronson, J.S. Pulskamp, R.G. Polcawich, C.M. Kroninger, and E.D. Wetzel, "PZT MEMS Actuated Flapping Wings for InsectInspired Robotics", IEEE Conf. MEMS, Sorrento, Italy, pp. 1047-1050, 2009.

[4] S. Dey, K. Budd, and D. Payne, "Sol-gel Processing of PbTiO3, PbZrO3, PZT, and PLZT Thin Films," Br. Ceram. Proc., vol. 36, pp. $107-121,1985.1$

[5] G. L. Smith, R. G. Polcawich, J. S. Pulskamp, Todd Waggoner, and J. F. Conley, Jr., "Atomic Layer Deposited Alumina for use as an Etch Barrier Against Xenon Difluoride Etching," Solid State Sensor, Actuator and Microsystems Workshop, Hilton Head Island, South Carolina, pp. 194-198, 2010.

[6] H. Chandrahalim, S. A. Bhave, R.G. Polcawich, J. Pulskamp, D. Judy, R. Kaul, and M. Dubey "Performance Comparison of $\mathrm{Pb}(\mathrm{Zr} 0.52 \mathrm{Ti0}$.48)O3-Only and $\mathrm{Pb}(\mathrm{Zr} 0.52 \mathrm{Ti0}$.48)O3-On-Silicon Resonators," App. Phys. Lett., vol. 93, pp. 233504 , 2008.

[7] S. S. Bedair, J. Pulskamp, B. Morgan, and R. Polcawich, "Performance model of electrode tailored thin film piezoelectric transformers for high frequency switched mode power supplies," Power MEMS 2009, Silver Spring, Maryland, pp. 435-438, December 1 - 4, 2009.

[8] D. J. Chung, R. G. Polcawich, D. Judy, J. Pulskamp, and J. Papapolymerou., "A SP2T and a SP4T Switch using Low Loss Piezoelectric MEMS,” IEEE MTT-S Microwave Symposium, pp. 21 24, June 2008.

[9] J.S. Pulskamp, D.C. Judy, R.G. Polcawich, R. Kaul, H. Chandrahalim, and S.A. Bhave, "Monolithically Integrated PiezoMEMS SP2T Switch and Contour Mode Filters", IEEE Conf. MEMS, Sorrento, Italy, pp. 900-903, 2009.

[10] J. S. Pulskamp, G. L. Smith, R. G. Polcawich, C. M. Kroninger, and E. D. Wetzel, "Two Degree of Freedom PZT MEMS Actuated Flapping Wings With Integrated Force Sensing," Solid State Sensor, Actuator and Microsystems Workshop, Hilton Head Island, South Carolina, pp. 390-391, 2010. 